\title{
A model to predict the residual layer thickness in the injection molding process
}

\author{
R. Mollaabbasi \\ Chemical engineering department \\ Université Laval \\ Québec, Canada \\ Roozbeh.mollaabbasi.1@ulaval.ca
}

\author{
E. Behzadfar \\ Husky Injection Molding Systems \\ Bolton, Canada
}

\author{
S. M. Taghavi \\ Chemical engineering department \\ Université Laval \\ Québec, Canada
}

\begin{abstract}
The effects of the wall temperature on the residual layer thickness of the polymers are studied numerically in the filling process in the injection molding process. A lubrication approximation model is developed to furnish a semi-analytical solution. The shear viscous heating terms are neglected to simplify the governing equations. The Cross-WLF viscosity model is used to capture the residual layer. There is a good agreement between the results of the developed model and experiments.
\end{abstract}

Keywords-Heat transfer; Cross-WLF model; non-Newtonian fluid; residual layer thickness; blocking time

\section{INTRODUCTION}

The mold filling process is the main stage in the injection molding process, which is the most common process in manufacturing plastic goods. During the filling process, a melted polymer fills the cavity part [1]. The temperature reduction due to the heat transfer of the mold with the walls during the filling process directly affects the viscosity of the polymer and, consequently, the filling flow rate that strongly influences the quality of the molded part [2]. The probability of the residual layer formation negatively affects the quality of the final products.

Several researchers have modeled the momentum and heat transfer in the injection molding process to find the velocity and temperature profiles [3, 4]. Newtonian [5], power-law [6, 7] and the Cross-WLF models [4, 8] have been used mainly to simulate the rheological properties of the polymers in the filling stage. The Cross-WLF model offers the best fit to the most viscosity data of polymers in the filling process [9] because the effects of shear rate and temperature on the viscosity of the polymers are considered simultaneously. The combination of heat-transfer analysis coupled with nonNewtonian fluid mechanics approach, also, can predict the velocity and temperature profiles of the polymer in the filling process [10].

Formation of the residual layer depends on the balance between the main forces that involve in the movement of fluid. In a different context, Allouche et al. [11] have investigated the residual layer in the displacement of two visco-plastic fluids in a plane channel. Their study quantifies the sufficient conditions for removing the residual layer basde on the value of the
Bingham number (as the ratio yield to viscous stresses). In general, the minimum and maximum thickness of the residual layer depends on the competition of yield stress and viscous stress $[12,13]$.

In this paper, we study the residual layer thickness following two scenarios, A. constant injection mass flux B. constant imposed pressure to simulate the formation and removing the residual layer in the filling process.

\section{MODELING}

The governing equations in the Cartesian coordinates $(\mathrm{x}, \mathrm{y})$ assuming a symmetric $2 \mathrm{D}$ channel flow in the dimensionless form are:

$$
\begin{aligned}
& \operatorname{Re}\left(\frac{\partial u}{\partial t}+u \frac{\partial u}{\partial x}+v \frac{\partial u}{\partial y}\right)=-\frac{\partial p}{\partial x}+\left[\frac{\partial}{\partial x} \tau_{x x}+\frac{\partial}{\partial y} \tau_{y x}\right] \\
& \operatorname{Re}\left(\frac{\partial v}{\partial t}+u \frac{\partial v}{\partial x}+v \frac{\partial v}{\partial y}\right)=-\frac{\partial p}{\partial y}+\left[\frac{\partial}{\partial x} \tau_{x y}+\frac{\partial}{\partial y} \tau_{y y}\right]+\frac{1}{S t} \\
& \frac{\partial u}{\partial x}+\frac{\partial v}{\partial y}=0 \\
& \left(\frac{\partial \theta}{\partial t}+u \frac{\partial \theta}{\partial x}+v \frac{\partial \theta}{\partial y}\right)=-\frac{1}{\operatorname{Re} \operatorname{Pr}}\left(\frac{\partial^{2} \theta}{\partial x^{2}}+\frac{\partial^{2} \theta}{\partial y^{2}}\right) \\
& -\frac{E c}{\operatorname{Re}}\left(\tau_{x x} \frac{\partial u}{\partial x}+\tau_{x y} \frac{\partial u}{\partial y}+\tau_{y x} \frac{\partial v}{\partial x}+\tau_{y y} \frac{\partial v}{\partial y}\right)
\end{aligned}
$$

Here, $u, v$ denote the dimensionless velocity components, $p$ is the pressure, $\tau$ represents the stress in the fluid. Prandtl, Reynolds, Stokes and Eckert numbers are defined as:

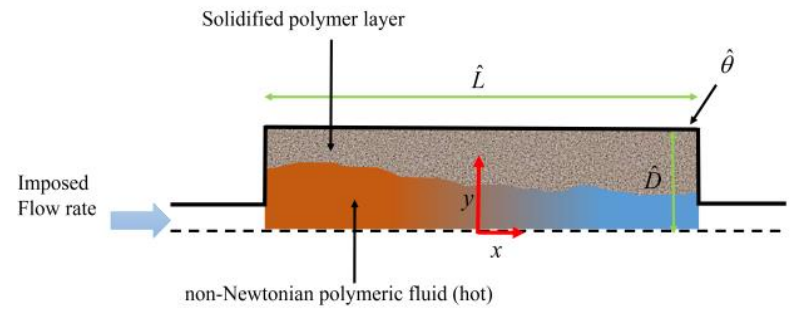

Figure 1. A schematic of problem. 


$$
\begin{aligned}
& \operatorname{Pr}=C \hat{p} \hat{\eta}_{00} / \hat{k}, \operatorname{Re}=\hat{\rho} \hat{u}_{0} \hat{D} / \hat{\eta}_{00}, \\
& S t=\hat{\eta}_{00} \hat{u}_{0} / \hat{\rho} \hat{g} \hat{D}, E c=\hat{u}_{0}{ }^{2} / C \hat{p}\left(\hat{\theta}_{i}-\hat{\theta}_{w}\right),
\end{aligned}
$$

where, $\hat{u}_{0}, \hat{D}, \hat{g}$ are the mean imposed velocity, the half depth of the cavity and the gravitational acceleration respectively. $C \hat{p}, \hat{k}, \hat{\theta}_{w}, \hat{\theta}_{i}$ are the specific heat capacity, the thermal conductivity, the wall temperature and the initial fluid temperature. $\hat{\eta}_{00}$ is the constant parameter of the Cross-WLF model. The dimensionless temperature is defined as $\theta=\left(\hat{\theta}-\hat{\theta}_{w}\right) /\left(\hat{\theta}_{i}-\hat{\theta}_{w}\right)$. We assume that the geometry is full of fluid when the molten polymer is injected to the cavity (see Figure 1). Based on the small aspect ratio of the problem ( $\delta=\hat{D} / \hat{L}$ ) we re-scale as follows:

$$
\delta x=X, \delta t=T, \delta p=P, \delta V=v .
$$

Applying the lubrication approximation $(\delta \operatorname{Re} \rightarrow 0)$ and neglecting the viscous dissipation terms, we can rewrite the governing equations as:

$$
\begin{aligned}
& \frac{\partial}{\partial y} \tau_{y X}=\frac{\partial P}{\partial X}, 0=\frac{\partial P}{\partial y}, \\
& \frac{\partial u}{\partial X}+\frac{\partial V}{\partial y}=0, \frac{\partial \theta}{\partial T^{*}}=\left(\frac{\partial^{2} \theta}{\partial y^{2}}\right),
\end{aligned}
$$

where, $T^{*}=T / \delta \operatorname{Re} \operatorname{Pr}$. The Cross-WLF model interprets the rheological properties of the polymer as;

$$
\begin{aligned}
& \tau_{y X}=\eta \dot{\gamma}, \eta=\frac{\eta_{0}}{1+\left(B \eta_{0} \dot{\gamma}\right)^{1-n}}, \\
& \eta_{0}=\exp \left(-\frac{A_{1}\left(\theta-\theta^{*}\right)}{A_{2}+\left(\theta-\theta^{*}\right)}\right),
\end{aligned}
$$

where, $\frac{\hat{\theta}_{w}-\hat{\theta}^{*}}{\hat{\theta}_{i}-\hat{\theta}_{w}}=-\theta^{*}, \frac{\hat{A}_{2}}{\hat{\theta}_{i}-\hat{\theta}_{w}}=A_{2}, \eta_{0}=\frac{\hat{\eta}_{0}}{\hat{\eta}_{00}}$,

$$
\eta=\frac{\hat{\eta}}{\hat{\eta}_{00}}, B=\frac{\hat{u}_{0}}{\hat{\tau}^{*}} \frac{\hat{\eta}_{00}}{\hat{D}} .
$$

$\hat{\eta}, \hat{\tau}^{*}, \hat{\eta}_{0}, \hat{\theta}^{*}, \mathrm{n}$ are the melt viscosity, the critical stress level at the transition to shear thinning, the zero shear viscosity, the glass transition temperature and the power law index respectively. $\hat{A}_{1}, \hat{A}_{2}$ and $\hat{\eta}_{00}$ are data-fitted coefficients.

\section{RESULTS AND DISCISSIONS}

Solving the momentum and energy equations considering the Cross-WLF model gives the velocity profile at different times. To calculate the temperature profile of the polymer along the filling process, we solve the energy equation $\left(\frac{\partial \theta}{\partial T^{*}}=\frac{\partial^{2} \theta}{\partial y^{2}}\right)$ analytically. Velocity profile obtained based on scenario A (constant injection mass flux) and scenario B (constant imposed pressure). The latter is to simulate the formation and removing the residual layer in the filling process.

\section{A. Constant mass flux}

In the first scenario, we considered the mass flux is constant along the process and the pressure drop is variable. We can then calculate the velocity profile during the filling process at different time.

Figure $2 \mathrm{a}$ and Figure $2 \mathrm{~b}$ demonstrate the temperature and velocity profiles of a polymer during the filling process versus time. The maximum temperature gradient exists at the beginning where the dimensionless melt temperature is 1 . It takes a finite amount of time for a temperature "wave" to propagate through the polymer and the melt temperature decreases. At longer times, the temperature gradient goes to zero and the polymer temperature becomes equal to the wall temperature.

The polymer layers close to the wall have small overall heat resistance and become cool faster in comparison to the layers close to the middle of the cavity. This is because the conduction heat transfer is the main mechanism in the heat transfer between the wall and melt in the filling process. Consequently, the viscosity of the polymer layers increase adjacent the wall and a residual layer appears. The temperature gradient decreases with time and the thickness of residual layer increases. The maximum velocity at the middle of the cavity increases with time since the mass flux needs to remain constant.

Figure 3 shows three different zones in which the residual layer thickness changes with time. In short times, the residual layer increases with time. Then the thickness decreases gradually and it becomes zero at longer times. This phenomenon can be explained by looking back at Cross-WLF model. The viscosity of the polymer depends on the temperature and the shear rate. In a short filling process, the temperature difference between the wall and polymer is considerable. Therefore, the viscosity of the polymer layer close to the wall increases dramatically and the residual layer thickness increases. Along the time, the temperature difference inside the polymer decreases and the shear rate effect increases. It removes the residual layer with time. In a long time process, the temperature gradient inside the polymer tends to zero and the shear rate is able to move the polymer completely.

The viscosity of the polymer increases by cooling the polymer. The pressure drop increases with time dramatically due to the constant mass flux in the filling process. The pressure drop becomes constant when the residual layer is removed completely. Note that in this stage the polymer temperature is close to the wall temperature; therefore, the viscosity does not change. 

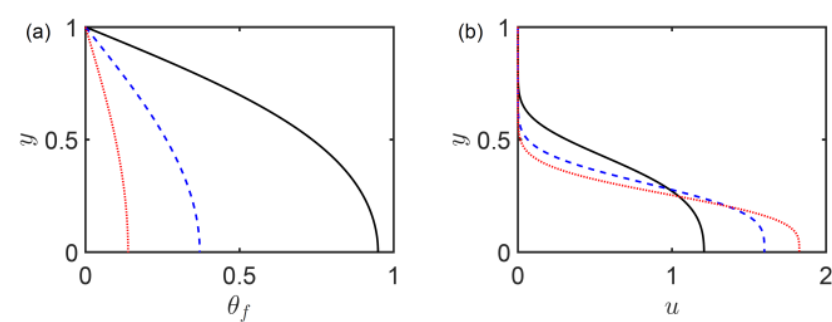

Figure 2. Temperature and velocity profiles at $T^{*}=0.1,0.5,0.9$ for the blacksolid, blue dashed and red dotted line. $A_{1}=31, \mathrm{~A}_{2}=0.2, \theta^{*}=0.12, \mathrm{n}=0.3, \mathrm{~B}=5 \times 10^{11}$.

\section{B. Constant imposed pressure}

The results of the previous section shows that the residual layer is removable at longer times if the pressure is increased dramatically. Looking back at Figure 3b, the imposed pressure must be increased almost ten orders of magnitude to remove the residual layer completely, which is not feasible for the industry. Therefore, we are considering the second scenario (constant imposed pressure) to understand the behavior of the residual layer during a more realistic filling process.

Figure 4 shows the velocity profile evolution of the polymer during the filling process in which the imposed pressure is constant. As we have discussed, the viscosity of the polymer increases due to the heat transfer between the wall and the melt. Therefore, the residual layer thickness increases with time in the case of constant imposed pressure (compare the residual layer in the inset of Figure $4 \mathrm{a}$ for $T^{*}=0.01$ (blue), 0.2 (black) and 0.4 (red)). In addition, increasing the viscosity of fluid causes the maximum velocity to decrease with time. Increasing the imposed pressure augments the driving force that enhances the maximum velocity (for instance compare the blue lines in the Figures $4 \mathrm{a}$ and $4 \mathrm{~b}$ ). In addition, higher pressure decreases the thickness of the residual layer. The dashed lines in the insets of Figures $4 \mathrm{a}$ and $4 \mathrm{~b}$ show the residual layer thickness reaching 0.52 and 0.48 at $P$ of $3 \times 10^{-12}$ and $4.2 \times 10^{-12}$ respectively when $T^{*}=0.4$.
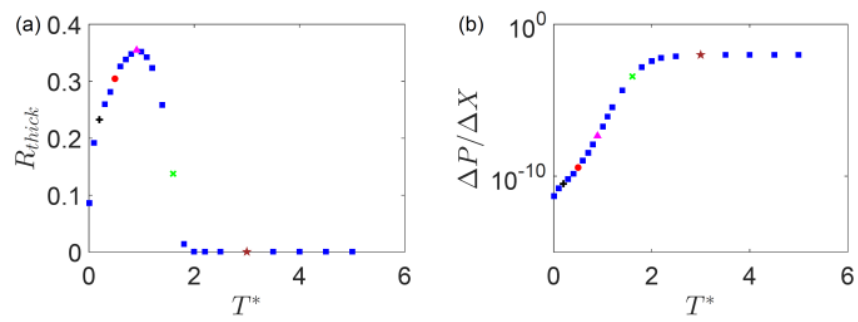

Figure 3. Dimenssionless residual layer thickness and pressure drop with time for $A_{1}=31, \mathrm{~A}_{2}=0.2, \theta^{*}=0.12, \mathrm{n}=0.3, \mathrm{~B}=5 \times 10^{11}$.
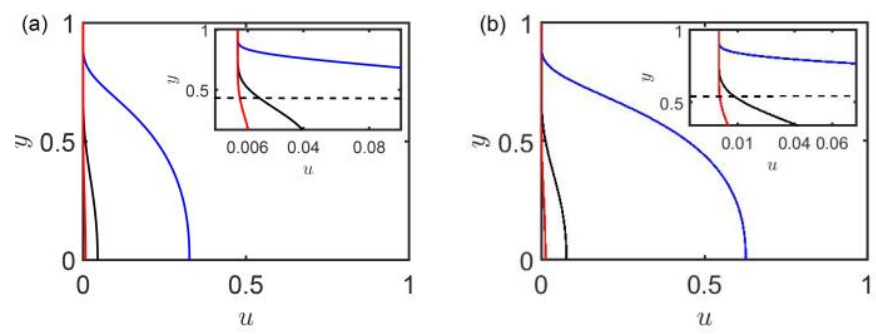

Figure 4. Velocity profile evolution along the time with the constant impose pressure. $A_{1}=31, \mathrm{~A}_{2}=0.2, \theta^{*}=0.12, \mathrm{n}=0.3, \mathrm{~B}=5 \times 10^{11}$. The imposed pressure of (b) is $40 \%$ greater than (a).

\section{Comparison with experiments}

In order to evaluate the developed model, a pilot tool was made to produce sheets with dimensions of $130 \mathrm{~mm} \times 30 \mathrm{~mm}$ $\times 3 \mathrm{~mm}$. A gate was located in the center of the cavity as shown in Figure 5. The cavity was equipped with two Kistler 61923 thermocouples flush with the surface and $44 \mathrm{~mm}$ apart from each other. The presence of the thermocouples enabled not only the measurement of the temperature of the cavity wall overtime, but also monitoring the advancement of the melt front inside the cavity. The response time of the thermocouples was $1 \mathrm{~m} / \mathrm{s}$. Using a thermolator, adjustments were made to the temperature of the cooling water and hence the cavity walls.

By controlling the injection pressure, we deliberately produced short shots to monitor the melt front advancement at different injection pressures. We used the traveling time of the melt front between two thermocouples to calculate the average velocity of the melt inside the cavity and calculate the blocking time (the time that the residual layer thickness becomes half of the cavity).

Table 1 shows the comparison between the blocking time coming from the present work, the model of Chen [14] and the experimental results. The model of Chen is an empirical method for the calculation of the blocking time. In this model, the blocking time is independent of the imposed pressure and depends on the thermal diffusivity, the melt temperature, the mold temperature and the no-flow temperature. Table 1 shows that there is a good agreement between the results of the present work and the experimental one.

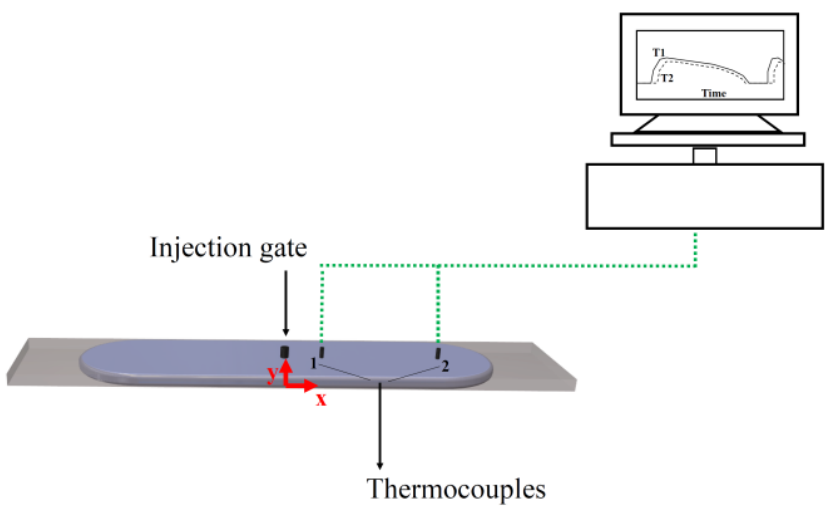

Figure 5. Schematic of the experimental setup. 
TABLE I. BLOCKING TIME COMPARISION

\begin{tabular}{|c|c|c|c|}
\hline \multirow{2}{*}[A_{2},-\theta^{*}]{} & \multicolumn{3}{|c|}{ Blocking Time } \\
\cline { 2 - 4 } & Experiments & Present work & Chen [14] \\
\hline$[0.19,0.08]$ & $0.725 \pm 0.10$ & 0.836 & 0.248 \\
\hline$[0.21,0.09]$ & $0.907 \pm 0.10$ & 0.915 & 0.215 \\
\hline$[0.28,0.11]$ & $0.915 \pm 0.10$ & 0.791 & 0.284 \\
\hline
\end{tabular}

\section{CONCLUSIONS}

Through developing a semi-analytical model, we have studied the residual layer that appear in the injection molding process. We have assumed that the geometry is full of the fluid when the molten polymer is injected to the cavity. We have relied on the Cross WLF to predict the rheological properties of the polymer. We have followed two scenarios when the momentum equation: Scenario A: constant mass flux; Scenario B: constant imposed pressure. The model is able to predict the blocking time of the cavity in the constant imposed pressure condition and there is a good agreement between the results of the model and experiments. Based on the developed model the residual layer disappears completely in the filling process if the imposed pressure increases several orders of magnitude in scenario A. However, this pressure is much higher than the pressure that is typically used in industrial cases.

\section{ACKNOWLEDGMENT}

This research has been carried out at Université Laval, supported financially by the NSERC Engage grant. The authors acknowledge Husky injection molding systems for the support of this work.

\section{REFERENCES}

[1] Z. Tadmor, C. Gogos, "Principles of polymer processing", John Wiley and Sons. 2013.

[2] J. Primo B-R, A. Dominguez-G, G. Herrera-Ruiz, M. Delgado-Rosas, "Filling process in injection mold: A review", Polym-Plast Technol 2007, 46, 721.

[3] M. Kamal, P. Laeur, "A structure oriented computer simulation of the injection molding of viscoelastic crystalline polymers, Model predictions and experimental results", Polym. Eng. Sci. 1986, 26, 103.

[4] B. Chen, W. Liu, "Numerical simulation of the post filling stage in injection molding with a two phase model", Polym. Eng. Sci. 1994, 34, 835.

[5] R. Chang, W. Yang, "Experimental and theoretical studies of shrinkage, warpage, and sink marks of crystalline polymer injection molded parts", Int. J. Numer. Methods Fluids 2001, 37, 125.

[6] M. Kamal, S. Kenig, "The injection molding of thermoplastics part I: theoretical mode", Polym. Eng. Sci. 1972, 12, 294.

[7] M. Barone, C. Tucker, Fundamentals of computer modeling for polymer processing, Hanser 1989.

[8] Y. Liu, "Heat transfer process between polymer and cavity wall during injection molding", 2014.

[9] C. Hieber, H. Chiang, "Shear-rate-dependence modeling of polymer melt viscosity", Polym Eng Sci 1992, 32, 931.

[10] T. Osswald, L.-S. Turng, P. Gramann, "Injection molding handbook", 2008.

[11] M. Allouche, I. Frigaard, G. Sona, "Static wall layers in the displacement of two visco-plastic fluids in a plane channel", J.F.M 2000, $424,243$.

[12] I. Frigaard, O. Scherzer, G. Sona, "Uniqueness and Non-uniqueness in the Steady Displacement of Two Visco-plastic Fluids", J. Appl. Math. Mech. 2001, 81, 99.

[13] I. Frigaard, S. Leimgruber, O. Scherzer, "Variational methods and maximal residual wall layers", JFM 2003, 483, 37.

[14] Z. Chen, A. Giacomin, L. Turng, "Flash", Polym Eng Sci 2006, 46, 241. 